\title{
La place des revues dans la communication scientifique en régime de libre accès
}

Retour sur le débat français en SHS

Ivan Jaffrin et Thomas Parisot

\section{OpenEdition \\ Journals}

Édition électronique

URL : http://journals.openedition.org/ress/2637

DOI : $10.4000 /$ ress. 2637

ISSN : 1663-4446

Éditeur

Librairie Droz

Édition imprimée

Date de publication : 6 mai 2014

Pagination : $9-35$

ISBN : 978-2-600-01829-6

ISSN : 0048-8046

Référence électronique

Ivan Jaffrin et Thomas Parisot, «La place des revues dans la communication scientifique en régime de libre accès », Revue européenne des sciences sociales [En ligne], 52-1 | 2014, mis en ligne le 12 mai 2014, consulté le 10 décembre 2020. URL : http://journals.openedition.org/ress/2637 ; DOI : https:// doi.org/10.4000/ress.2637 


\title{
LA PLACE DES REVUES DANS LA COMMUNICATION SCIENTIFIQUE EN RÉGIME DE LIBRE ACCÈS RETOUR SUR LE DÉBAT FRANÇAIS EN SHS
}

\author{
IVAN JAFFRIN \& THOMAS PARISOT \\ Revue européenne des sciences sociales (Librairie Droz) \& Cairn.info \\ ivan.jaffrin@droz.org/thomas.parisot@cairn.info
}

\begin{abstract}
Résumé. En guise d'introduction au dossier que publie la Revue européenne des sciences sociales sur les enjeux du libre accès en sciences humaines et sociales, le présent article revient sur la polémique qui a suivi, en France, dans le secteur des SHS, la publication de la Recommandation de la Commission européenne du 17 juillet 2012 relative à «l'accès aux informations scientifiques et à leur conservation ». Après avoir observé les argumentations en présence et analysé les stratégies des différents acteurs impliqués, les auteurs s'efforcent d'entrevoir ce que pourrait être l'avenir de la communication scientifique en régime de libre accès, à l'aune de de deux grands paradigmes («légal-bibliométrique» vs «collaboratif-en-réseau»). Ce faisant, ils s'interrogent sur le rôle des revues traditionnelles en SHS dans une économie de l'attention et s'alarment de quelques-unes des conséquences indésirables d'une communication scientifique faisant la part belle aux données et laissant aux seuls algorithmes ou réseaux sociaux la fonction de prescrire de la pertinence.
\end{abstract}

Mots-clés: bibliométrie, communication scientifique, économie de l'attention, libre accès, méga-revues, revues scientifiques, réseaux sociaux, sciences humaines et sociales.

\begin{abstract}
As an introduction to this section dedicated to Open Access in Human and Social Sciences by the Revue européenne des sciences sociales, this article deals with the debate spurred in France by the publication of the European Commission Recommandation about "access to and preservation of scientific information". After analysing the arguments and strategies of the different stakeholders involved, the authors sketch out how an open access scientific communication may look like with regard to two dominant paradigms ("legal-bibliometrical" vs "collaborativenetworking"). In doing so, they question the role of HSS journals in the context of the economy of attention and put the focus on the unexpected consequences that may derive from a scientific communication based on data and relying on the prescription operated by algorithms and social networks.
\end{abstract}

Keywords: academic journals, bibliometry, economy of attention, human and social sciences, mega-journals, open access, scholarly communication, social networks. 


\section{LE LIBRE ACCÈS À L'INFORMATION SCIENTIFIQUE EN DÉBAT Registres argumentatifs et stratégies d'acteurs}

\section{I.I.RAPPEL DU CONTEXTE'}

La publication d'une Recommandation de la Commission européenne (CE), le I7 juillet 20I2, relative à «l'accès aux informations scientifiques et à leur conservation» (20I2a), est à l'origine d'une controverse en France quant à son application dans le domaine des sciences humaines et sociales (SHS), et quant au préjudice qu'elle pourrait porter au système éditorial des revues. [Pour un point de vue plus développé sur ladite Recommandation, voir l'article de Xavier Landes, dans ce dossier, section 4.]

À l'issue d'une rencontre entre représentants de revues scientifiques et éditeurs, organisée le i I février 20ı3 à l'initiative du portail Cairn.info, une motion (20I3a) a été signée par plus de I20 revues francophones s'inquiétant d'une application stricte de la Recommandation de la CE sur l'économie des revues et appelant à une concertation de tous les acteurs ainsi qu'à la réalisation d'une étude d'impact préalable. Consécutivement à cet appel, une tribune a paru dans le journal Le Monde intitulée «Qui a peur de l’Open Access ? (20 zb), signée par un grand nombre d'enseignants-chercheurs et membres de la communauté universitaire, largement relayée, cette fois-ci, par la plate-forme OpenEdition, qui a notamment en charge le portail Revues.org. Il s'agit a contrario, pour ces derniers, de faire valoir l'opportunité que constituerait le libre accès pour les SHS fran-

I La Revue européenne des sciences sociales (Ress), au nom de son comité de rédaction, est signataire de la motion du II février, intitulée «Open Access: le travail scientifique en sciences humaines et sociales et le débat public fragilisés par les mesures préconisées par la Commission européenne». Éditée par la Librairie Droz (Genève), la Ress bénéficie d'un contrat tripartite qui lui permet d'être diffusée simultanément par le portail Cairn.info (pour la partie payante, correspondant aux trois dernières années de publication) et par le portail Revues.org (pour les numéros antérieurs à trois ans). Elle est à ce titre particulièrement concernée par le débat qui s'est fait jour et ne pouvait manquer d'y prendre part. En ouverture au dossier sur les enjeux du libre accès en SHS que publie la Ress, les auteurs du présent article - respectivement secrétaire de rédaction de la Ress à la Librairie Droz et responsable des relations institutionnelles chez Cairn - entendent poser de manière générale les conditions préalables qui leur semblent devoir être réunies pour qu'une délibération équitable puisse avoir lieu en connaissance de cause. S'exprimant ici en leur nom propre, ils espèrent pouvoir étayer les motivations qui ont poussé un grand nombre de revues, dont la Ress, a signer l'appel lancé par Cairn. 
cophones dans la perspective d'un grand mouvement de démocratisation de l'accès au savoir et d'innovations éditoriales.

Un tel débat est largement redevable, précisons-le, d'un contexte qui voit la plupart des institutions publiques de la recherche, nationales ou supranationales, faire montre d'un grand volontarisme dans la mise de l'Open Access (OA) à l'agenda des politiques publiques. À l'occasion des journées Couperin de janvier 2013, la ministre de l’Enseignement supérieur et de la Recherche, Geneviève Fioraso, avait ainsi réaffirmé l'engagement de la France en faveur du principe du libre accès ainsi que son soutien aux archives ouvertes, tout en appelant à explorer toutes les autres voies possibles. Quelques mois plus tôt, les conclusions du rapport Finch «Accessibility, sustainability, excellence: how to expand access to research publications » (20I2), paru au Royaume-Uni, avaient été avalisées par le gouvernement britannique, non sans susciter, là aussi, une vive controverse. En février 20ı3, c'était au tour des États-Unis d'adopter un mémorandum publié par l'Office of Science and Technology Policy en faveur de l'accès public aux résultats scientifiques financés par l'État fédéral. C'est ensuite l'organisme Science Europe qui rendit public en avril de la même année une série de principes visant à assurer la transition vers l'OA. Enfin, les membres du G8 ont exprimé en juin 2013 leur engagement commun à rendre accessibles les données publiques et à s'efforcer d'accroître le libre accès aux résultats scientifiques. [Ghislaine Chartron revient sur cette actualité dans l'article qu'elle publie dans ce dossier, sections I.2 et I.3.]

Quant à la Recommandation de la CE, elle fait elle-même suite à une série de rapports et de communiqués sur l'information scientifique à l'ère numérique ayant largement ouvert la voie aux politiques de libre accès, dans une double perspective de valorisation des investissements de recherche européens et de soutien à l'innovation. Ainsi prend-elle place dans le cadre d'une stratégie globale, dite «Horizon 2020 », pour l'innovation et la croissance en Europe, qui porte à près de 80 milliards d'euros le budget consacré à la Recherche \& Développement, et fait une place considérable aux enjeux de diffusion et préservation numérique des données scientifiques. Et pour cause: «le libre accès aux résultats de la recherche stimulera la capacité d'innovation en Europe» est-il affirmé dans un communiqué (CE, 20I2b). Ladite Recommandation s'inscrit à cet égard 
également dans la lignée d'un précédent rapport de l'OCDE sur l'économie de l'information qui insistait déjà, en 2005, sur le rôle des publications scientifiques dans la dissémination de la connaissance et préconisait l’adoption de mesures visant à augmenter le rendement des investissements publics en R\&D de manière à améliorer les capacités d'innovation de nos économies². [Sur les croyances associées au libre accès en matière d'innovation et de croissance, on peut se reporter à l'article de Hans Dillaerts, dans ce dossier, sections I.I, 2.3 et conclusion.]

\section{I.2. ARGUMENTATION ET ENJEUX}

D’innovation et de croissance, il n’a cependant guère été question à l'occasion du débat qui s'est fait jour autour des enjeux du libre accès en SHS. Et le contenu exact de la Recommandation de la CE - pourtant à l'origine des inquiétudes exprimées par les signataires de la motion du i février - n’a pas non plus fait l'objet d'un examen approfondi. Il s'est agi bien plutôt d'affirmer avec plus ou moins de vigueur son adhésion aux principes de l'OA, et par voie de conséquence de s’afficher comme partisan ou non d'une «démocratie du savoir» idéale.

Peut-être convient-il de rappeler, à toutes fins utiles, que la non-adhésion au principe de l'OA, tel qu'il a été formulé par la CE, ne saurait valoir ipso facto adhésion à un principe strictement inverse, de nature a priori non-démocratique. Le terme même d'«open» ou de «libre », s'agissant de l'accès aux résultats de la recherche publique, souffre en effet peu la contestation: sauf à se faire le chantre d'un accès fermé par principe - ce qui, on en conviendra, n'est pas une proposition acceptable -, il apparait difficile d’en contester la légitimité. Et de fait, il ne s'est trouvé guère de monde pour dénoncer l'OA dans son principemême, au point qu'un certain de nombre de revues signataires de la motion du I février l'ont été également de la tribune du Monde.

2 À propos du contenu des publications de l'OCDE, on pourra avantageusement se référer à Vajou et al., 2009. Notons encore que l'OCDE a publié par la suite une série de principes et lignes directrices «pour l'accès aux données de la recherche financée sur fonds publics» (2007). 
L'emploi de tels «mots-valeur» autorise un déplacement de l'énonciation sur le terrain de la morale à travers l'expression de jugements normatifs, a priori irréfutables. Pareils énoncés ne sauraient cependant être valides sans un accord préalable de toutes les parties sur la définition du bien commun à l'aune duquel on entend se placer. Si l'on veut bien accepter ce parallèle avec les débats qui ont suivi, en France, l’adoption de la loi relative aux Libertés et Responsabilités des Universités (LRU), il apparaîtra sans doute évident que la valeur «autonomie», dans le contexte d'une loi réformant le statut des universités, ne saurait être un bien commun unanime si elle devait se traduire par une crise structurelle des budgets des universités, une mise en concurrence des unes avec les autres et, pour finir, par à un renoncement progressif à ce qui fait leur raison d'être : la formation universitaire ${ }^{3}$. La question est évidemment bien plutôt celle des moyens et des dispositions concrètes permettant à une valeur reconnue par tous de se traduire dans les faits. On ne saurait en conséquence imputer a priori à ceux qui s'inquiètent de la manière dont une politique publique en faveur du libre accès va être menée d'être «fermés», ou de ne pas assez aimer l'«ouverture », au motif qu'ils n'adhèreraient pas à l'impératif de se conformer à des mutations présentées d’emblée comme bonnes, nécessaires et inéluctables ; que ce soit au nom du «tournant Web», de la compétition internationale, de la transparence ou encore de l'efficience de la recherche.

Un tel «sophisme du changement», outre qu'il a été abondamment utilisé à l'encontre des adversaires de la LRU, avec les résultats qu'on sait, pose un jugement de valeur implicite qui ne peut être accepté sans discussion. L'unanimité apparente en faveur du libre accès masque en effet une pluralité de registres argumentatifs, éventuellement contradictoires, visant à fonder en légitimité l'adhésion à l'OA. Il résulte de cette relative polyphonie des discours non seulement une incertitude globale quant à la «valeur» sur la base de laquelle on est appelé à juger, mais, plus encore, un flou permanent sur la finalité de l'opération et le type de dispositifs à mettre en œuvre pour l'accomplir concrètement.

3 Voir à ce sujet Nathalie Brafman, 2013, «Les universités multiplient les plans de rigueur», Le Monde, 17 juillet: <http://lemonde.fr/enseignement-superieur/article/2013/07/16/lesuniversites-multiplient-les-plans-de-rigueur_3448I66_|473692.html>. 
Pour les uns, les tenants traditionnels de l'OA, le libre accès est ainsi avant tout affaire de partage et de dissémination de la recherche. Il s'agit de donner les moyens aux chercheurs de s'affranchir des intérêts économiques en jeu dans la publication scientifique en se réappropriant les canaux de la diffusion scientifique et en s'émancipant des grands groupes internationaux de presse et d'édition. Tel n'est cependant pas l'objectif premier des institutions publiques ou des bailleurs de fonds qui soutiennent le libre accès, et en l'espèce de la $\mathrm{CE}$, dont la motivation repose avant tout sur la recherche de l'efficience de l'investissement public et de l'innovation. Pour d'autres encore, le libre accès vise essentiellement à réparer une iniquité fondamentale et doit s'entendre d’abord comme la revendication d'une égalité d’accès de tous à tous les résultats de la recherche, que l'on soit chercheur ou simple citoyen, «amateur éclairé» ou «lecteur inattendu», riche ou pauvre, du centre ou de la périphérie, du Nord ou du Sud, etc.

Ce faisant, chacun prétend se placer sous l'intérêt général ou, en l'occurrence, le progrès scientifique, seul bien commun unanime dans la discussion. Et pour cause, toute argumentation visant une autre finalité que le progrès de la science, ou s'affirmant en contradiction avec elle, transgresserait l'« ethos scientifique » même. Il n'en demeure pas moins que les moyens d'y parvenir diffèrent sensiblement selon le registre argumentatif que l'on promeut et le type de public auquel on s'adresse. S’agit-il de promouvoir l'efficience de la recherche à travers la mise en place d'instruments de mesure ou bien de combler une injustice dans l'accès à l'information scientifique? Entend-on par là faire primer le principe de l'accès à tous aux résultats de la recherche indépendamment des conditions de leur production et de leur diffusion, ou bien faut-il comprendre que le libre accès est au contraire le moyen d'une réappropriation par les chercheurs eux-mêmes des canaux de la diffusion scientifique ? Et pourra-t-on in fine accorder entre elles logique de marché, revendication citoyenne et autonomie des chercheurs sur la seule foi du libre accès? [Les enjeux normatifs et politiques de l'OA sont abordés en conclusion de l'article de Xavier Landes, dans ce dossier.] 


\section{I.3. STRATÉGIES ET INTÉRÊTS DES ACTEURS}

S’ajoute à la pluralité des registres d’argumentation en présence et au caractère manifestement non-unanime de la définition du bien commun qu'implique la notion de «libre accès», une diversité de stratégies d’acteurs visant chacune à conformer les politiques en faveur de l'OA à leurs missions et intérêts respectifs. [Pour un panorama complémentaire des intérêts en jeu dans la diffusion de la recherche, on peut se reporter à l'article de Xavier Landes, dans ce dossier, section I.]

\section{I.3.I.Les bailleurs de fonds: États, agences, Commission européenne}

Que ce soit par l'intermédiaire d'organismes nationaux en charge de la politique de la recherche et de son financement ou, plus directement, par le truchement de la loi, les États ont définit progressivement leur position en matière de libre accès aux résultats de la recherche, pour partie collectivement (comme récemment dans le cadre du G8), mais en règle générale de manière non-coordonnée.

Certains ont fait le choix, comme au Royaume-Uni, d'axer principalement leur politique sur le financement en amont des publications scientifiques: le rapport Finch privilégie ainsi explicitement (soutiens financiers à l'appui) les modèles de type «auteur-payeur» (voir Finch, 20I2 et RCUK, 20I3) au grand dam d'une partie de l'opinion scientifique. On notera cependant que les pouvoirs publics britanniques sont par la suite partiellement revenus sur les conclusions du rapport en ménageant des options de publication alternatives, de manière, notamment, à faire face à la complexité et au coût de la transition amorcée.

D’autres États, au contraire, à l'image de l'Allemagne, se sont résolument orientés vers l'auto-archivage des articles scientifiques par leur(s) auteur(s), au sein de répertoires institutionnels ${ }^{4}$.

Quant à la position française, elle est à ce jour encore hésitante, et se borne à prendre acte de la complexité du débat en appelant à raisonner au niveau de chaque discipline et à développer le dialogue entre les parties prenantes.

4 Voir «Point sur le Libre Accès en Allemagne», INIST: <http://openaccess.inist.fr/?Point-surle-Libre-Acces-en>. 
Par delà la diversité des politiques mises en œuvre par les États, un point est en tout cas récurrent, même s'il n'est pas toujours explicite : le lien entre ce débat et celui portant sur l'évaluation de la recherche, que celle-ci soit perçue comme un levier utile, voire indispensable, à la promotion des archives ouvertes ${ }^{5}$, ou qu'on considère sa généralisation comme inhérente au développement du libre accès. C'est en effet l'un des corollaires du développement de grandes bases de données scientifiques en libre accès que de permettre la mise en place sui generis d'indicateurs alternatifs pour la gestion des carrières, conformément aux vœux-mêmes de la CE.

\section{I.3.2. Les bibliothèques}

L'essor du numérique et son affirmation progressive comme vecteur principal de diffusion des publications scientifiques participent d'un bouleversement des missions des professionnels de la documentation, à un point tel qu'on pourrait certainement parler aujourd'hui d'une véritable crise identitaire du métier de bibliothécaire.

Les modèles de «Big Deals » développés pour la commercialisation des publications scientifiques au format numérique, initialement fondés sur une recherche de recouvrement des coûts fixes importants - induits par le développement des infrastructures de services numériques - et donc sur la réalisation d'impérieuses économies d'échelle ${ }^{6}$, ont en effet donné lieu à des modalités de négociation de plus en plus dures entre bibliothèques et éditeurs pour l’accès aux publications jugées essentielles par les publics universitaires. Or comme le souligne à juste titre Karine Delvert (2012) dans

5 Un «bâton» même, pour reprendre l'expression du recteur-président de l'université de Liège, Bernard Rentier, qui s'exprimait sur ce sujet lors des journées Couperin de janvier 2013. Les archives ouvertes concourent en effet au développement d'instruments de pilotage permettant la mesure de la productivité des chercheurs et des laboratoires, tout en entendant assurer à leurs travaux une plus grande et plus immédiate audience (quitte à attribuer une distinction aux plus téléchargés d'entre-eux, à la faveur d'un «hit parade mensuel», selon les propos de Bernard Rentier: voir <http://webcast.in2p3.fr/videos-conclusions_harnad> [24e minute]).

6 Contrainte économique qui, remarquons le au passage, vaut tout aussi bien pour les ressources en «accès conditionnel» que pour les ressources en «accès libre» (Schopfel, et al., 20 I2), en poussant structurellement à des formes de concentration dans les deux cas. 
son étude menée auprès de bibliothécaires français, l’activité de négociation ne relève pas du cœur de métier des bibliothécaires et est souvent peu valorisée au sein de la profession. L'OA suscite ainsi d'autant plus l'engouement des bibliothèques qu'il est porteur d'une reconversion possible de leur mission au sein de l'économie de la publication scientifique numérique, plus conforme à ce qu'elles considèrent être leurs prérogatives essentielles.

Parallèlement, la dépossession grandissante des missions de conservation traditionnellement assurées par chaque bibliothèque au profit de tiers-archiveurs nationaux et internationaux ${ }^{7}$, mais surtout au bénéfice des portails d'éditeurs ou d'agrégateurs assurant la diffusion numérique des publications (que ce soit à titre gratuit ou payant), prive progressivement les bibliothèques de ce qui constituait pour elles une forme d'identité : leurs collections. Le souhait d'une réappropriation des collections par les bibliothèques pousse là aussi ces dernières à préférer des modèles où, comme dans le cas de l'auto-archivage des publications par leurs auteurs sur les serveurs des institutions et leur diffusion gratuite à partir de ceux-ci, elles restent au cœur du processus de conservation.

On pourrait ajouter un dernier phénomène qui interroge fondamentalement le rôle des bibliothèques universitaires en tant que lieu physique de consultation et d'archivage, et accroît chez elles la tentation d'un changement radical de paradigme : la baisse continue de leur fréquentation par les publics de chercheurs et la nécessité de se repositionner vis-à-vis de ce public. Ce faisant, elles voient dans l'OA l'occasion «d'occuper une place centrale dans la diffusion des connaissances en assumant un rôle d'éditeur» (Malotaux, 20I I).

\section{I.3.3. Les éditeurs}

Parallèlement au développement des revendications pour le libre accès, les éditeurs de SHS de l'espace francophone font face à une très sensible dégradation du secteur de la publication de savoir: recul lent mais continu des achats de livres, singulièrement dans le cas d’ouvrages réputés exigeants (dans le

7 Constat relayé récemment par la «Bibliothèque Scientifique Numérique», constituée à l'initiative du ministère de l'Enseignement supérieur de la Recherche: <http://www.bibliothequescientifiquenumerique.fr/?BSN-6-Archivage-perenne>. 
domaine de l'édition en histoire, Sophie Barluet [2005] établissait il y a une dizaine d'années déjà que le marché avait été divisé par deux en 30 ans), étiolement du réseau des librairies de premier niveau - qui constitue traditionnellement le vecteur privilégié des ouvrages à rotation lente -, repli de la langue française dans le monde académique et au-delà, évolution des pratiques et des attentes des lecteurs au profit d'une logique d'immédiateté, etc. En conséquence, la situation économique des structures éditoriales françaises ayant en charge des publications en SHS (qu'elles soient publiques ou privées) est aujourd'hui très délicate (l'amalgame avec les grands groupes internationaux, dont les performances économiques sont souvent pointées du doigt, étant à ce niveau absolument sans fondement ${ }^{8}$ ) et c'est dans ce contexte que l'injonction à se conformer à l'OA leur est donnée. [Les caractéristiques structurelles des revues en SHS sont présentées par Ghislaine Chartron dans l'article quelle publie dans ce dossier, section 2.2.]

La journée organisée par Cairn sur cette question a été l'occasion de fournir quelques éléments susceptibles de préciser l'équation que les structures éditoriales françaises devront progressivement résoudre : la vente de licences d'accès aux universités françaises représente aujourd'hui un chiffre d’affaires annuel de I,5 millions d'euros hors taxes, montant réparti entre les presque 300 revues qui composent aujourd'hui les bouquets Cairn proposés aux universités françaises (portées par un peu plus de 120 structures éditoriales, dont 60\% environ sont publiques ou associatives). Dans un billet que la revue Tracés a mis en ligne le 8 mars 20ı3, la rédaction de la revue indiquait:

Dans le cas de Tracés, qui publie 3 volumes par an et qui bénéficie du soutien d'ENS Éditions, I5 ooo euros par an environ suffiraient. Étant donné le nombre élevé de revues, le montant total des aides pourrait cependant se révéler irréaliste. Mais on peut très bien considérer qu'il ne s'agit là que d'un problème de coordination, car le montant total versé chaque année par les bibliothèques et organismes publics de recherche français pour accéder à Tracés est sans doute bien supérieur (Tracés, 2013).

8 Pour ce qui est des stratégies des grands groupes d'édition dans le domaine des sciences de la vie, des sciences exactes ou de la médecine, on peut se reporter au dossier efficace et synthétique de la revue Nature (2013). 
Or toute la difficulté est bien là. Dans le cas des publications en SHS, il ne s'agit de toute évidence pas d'un problème de coordination mais bien d'une question structurelle de financement: l'extrapolation du besoin en financement indiqué par la revue Tracés pour, par exemple, les 400 revues diffusées sur le portail Cairn.info nous conduit à un montant de 6 millions d'euros, soit 4 fois plus que la contribution actuelle des bibliothèques et organismes publics de recherche français au fonctionnement des revues de SHS de langue française. Notons par ailleurs que ce calcul rapide ne tient compte ni des diversités de situation de chaque revue - qui devraient faire l'objet d'études précises (comme l'appellent d'ailleurs de leurs vœux les signataires de la motion Cairn comme ceux de la tribune du Monde) - ni des coûts d’acteurs qui, comme Cairn ou OpenEdition/Revues.org, œuvrent à la diffusion des contenus.

Au-delà même de ces enjeux de financement qui restent somme toute modestes au regard des budgets français de l'Enseignement supérieur et de la Recherche, ou même des budgets annuels consacrés par les bibliothèques à l'acquisition ou à la valorisation de la production scientifique, par exemple, les questions que pose la refondation du mode de financement de la publication en SHS sont nombreuses et demandent de toute évidence un véritable examen de politique scientifique avant toute considération stratégique. [Voir à ce sujet l'article de Ghislaine Chartron, dans ce dossier, section 4.]

\section{L'AVENIR DE LA COMMUNICATION SCIENTIFIQUE EN RÉGIME DE LIBRE ACCÈS Quelques hypothèses pour les SHS}

\section{I. LES DEUX «VIRTUALITÉS »DE L'OPEN ACCESS}

À la pluralité des argumentations et à la diversité des stratégies d’acteurs en matière d'OA - traduisant des intérêts manifestement divergents -, s'ajoute une incertitude générale quant à ce dont on parle, en terme de dispositifs concrets et de finalités opérationnelles, lorsqu'on invoque le libre accès aux résultats scientifiques. Par delà l'extrême complexité des modalités d’implémentation 
d'une politique de libre accès ${ }^{9}$, et le subtil raffinement entre les différentes «couleurs» (green, gold, platinium) et autres «saveurs» (free, libre, gratis) qui prévalent dès lors qu'il s'agit d’aborder la question de l'OA - prénotions qui tendent au demeurant à priver les non-initiés de la capacité à intervenir dans le débat -, il nous apparaît possible d'en formuler les enjeux en nous affranchissant des caractéristiques techniques habituelles.

Ce que donne à voir le débat sur l'OA n'est ainsi pas sans rappeler une opposition récurrente dans l'environnement numérique entre, d'une part, un modèle d'intégration verticale - à l'image des dispositifs d'«offre légale » de catalogues de titres numériques promus dans certains secteurs d’activités (et qui supposent le choix d'un modèle économique : l'acte d'achat, le forfait, la publicité, l'impôt, etc.) -, et, d'autre part, un modèle d'intégration horizontale fondé sur une économie du partage, faisant fi des intermédiaires, et qui trouve son expression la plus parfaite dans l'échange «peer-to-peer $»^{10}$.

Ces deux schémas s'inscrivent dans le débat qui nous occupe comme deux grandes «virtualités» de l'OA. Elles tiennent lieu d'utopies documentaires rivales, ou d'idées directrices concurrentes, dont le caractère non formulé peut s'avérer source de malentendus persistants. Il n'en demeure pas moins que, poussées à leur terme logique, elles attestent l'une et l'autre du fait que le libre accès ne saurait signifier simplement l'«ouverture » à ce qui était auparavant «fermé», par le passage du payant au gratuit, sans que le contenu de ce à quoi on accède - ou son environnement - ne s'en trouvât modifié aucunement. Il induit au contraire des mutations profondes quant à la nature du contenu et des services qui l'accompagnent; mutations portées par l'infrastructure socioéconomique et technologique qui rend possible le libre accès.

9 Voir à ce sujet le billet de David Wojick du II novembre 20 I3, sur le blog The Scholarly Kitchen: «Open Access on the Sea of Confusion»: <http://scholarlykitchen.sspnet.org/2013/1 I/II/ open-access-on-the-sea-of-confusion/>.

I0 Précisons d'emblée qu'il va de soi que l'échange peer-to-peer dans le cas de la publication scientifique ne relève pas dans notre propos d'un acte nécessairement délictueux par rapport à une offre réputée légale, mais seulement d'une des modalités possibles de la communication scientifique dès lors qu'elle n'est pas en infraction avec les droits cédés à un éditeur, que celui-ci soit public ou privé. 
Dans l'hypothèse d'une généralisation d'un régime d'accès de type vertical, on pourrait alors assister à l'établissement d'une vaste bibliothèque à guichet unique, adossée à des services bibliométriques et de text/data mining d'autant plus performants qu'ils s'effectueraient sur une masse exhaustive et homogène de documents. Un tel régime de libre accès reviendrait à institutionnaliser une forme de dépôt légal numérique et d’archivage pérenne centralisé de la version «officielle», telle que publiée en revue, sans que cela modifie substantiellement le protocole traditionnel de la publication scientifique. En ce sens, il s'accommoderait aussi bien des licences de type Creative Commons que du régime du droit d'auteur traditionnel. Il serait en outre conforme à la vision de l'OA au sens strict (accès gratuit et immédiat au dernier état du document publié), tout en maintenant intact le statut de la chose publiée et en préservant l'intégrité de la notion d’œuvre, par rapport au document «non publié» et à toute l'étendue de la littérature grise.

La véritable révolution d'un tel modèle procéderait alors de la systématisation des mesures d'évaluation bibliométrique article par article (statistiques de consultation, citations, téléchargements, etc.), rendue possible par la technologie numérique et l'effet d'échelle. Il en résulterait une puissante intégration des SHS, dont l'ensemble des publications pourrait être soumis à une même logique de description et de classification, chacune se voyant possiblement attribuer un rang ou un score, selon leur catégorie ou leur discipline d’appartenance. Ce paradigme que l'on pourrait appeler «légal-bibliométrique »" aurait très certainement les faveurs des agences d'évaluation et des bailleurs de fonds, en ce qu'il permettrait le développement d'indicateurs alternatifs en lieu et place du facteur d'impact des revues, dont l'importance en sciences techniques et médicales (STM) fait l'objet de critiques de plus en plus fortes. Dans le cas d'espèce des SHS - où la mesure du facteur d'impact apparaît moins légitime et est de fait moins répandue qu’en STM -, ce schéma viendrait même combler opportunément un vide en matière d'évaluation de la performance de la recherche publique, en fournissant un instrument de mesure quantitatif qui faisait jusqu’à présent défaut.

II Nous entendons «légal», non pas au sens de ce qui est conforme à la loi, par opposition à ce qui ne le serait pas, mais en ce que ce modèle emprunte la forme traditionnelle du droit pour faire le départ entre la «chose publiée» et tout ce qui ne l'est pas. 
Il va de soi qu'une telle vision des sciences sociales, soumises à l'impératif d'évaluation et aux mesures bibliométriques, est sujette à caution. Sans contester la nécessité et la légitimité du principe même de l'évaluation de la recherche, le moins que l'on puisse dire est que de telles mesures bibliométriques, si elles constituent sans doute un progrès par rapport au règne sans partage du facteur d'impact, n'en sont pas moins susceptibles de biais nombreux et d'une instrumentalisation à des fins de classement qui ne sauraient laisser indifférents les chercheurs en sciences sociales soucieux de leur indépendance. Qu’adviendrait-il de pans entiers de la recherche dans le secteur des humanités, ou dans des domaines relevant d'une très grande spécialisation, si l'on devait s'en remettre à ce type d'instruments de mesure pour orienter l'allocation des crédits de recherche? Et si telle devait être l'une des conséquences inévitables d'une politique d'OA, cela ne devrait-il pas faire l'objet d'une concertation préalable?

Une toute autre vision du régime de la publication scientifique émanerait de l'hypothèse de la généralisation de l'auto-archivage et d'un mode de diffusion horizontal de type «peer-to-peer $»^{{ }^{2}}$. Il s'agirait alors moins d'aboutir à une base documentaire homogène, fortement intégrée et gérée comme un instrument d'évaluation de la recherche, que de mettre en place un espace de communication autorégulé et non médiatisé entre chercheurs. Une telle vision est d’ailleurs parfaitement conforme aux potentialités du Web au sens où elle privilégie le principe de dissémination maximale des résultats de la recherche au détriment des conventions éditoriales existantes. Elle procède d'un paradigme que l'on pourrait appeler «collaboratif-en-réseau», dans la mesure où l'inclusion dans une communauté prime sur la logique de différenciation entre contenu «publié» et littérature grise. Loin d'être l'aboutissement d'une recherche, l'article y est conçu comme un moment du dispositif scientifique, non définitif, ouvert aux commentaires et qui n'est qu'une forme voisine du billet de blog ou du carnet de recherche.

12 À l'exemple du site AcademicsTorrent, plate-forme explicitement «peer-to-peer» de partage des données scientifiques: <http://academictorrents.com/>. 
Nous assisterions alors à une révolution bien plus profonde du régime de la publication scientifique, telle que la chose publiée perdrait son caractère consacré à la faveur d'un éclatement des formats. L'article de recherche viendrait ainsi côtoyer des niveaux de granularité subsidiaires et existerait sous la forme de versions successives, comme un continuum d'unités documentaires. En lieu et place d'une bibliothèque unique et centralisée, pourrait alors prendre forme un espace de la communication scientifique où chaque unité documentaire, de quelque niveau de granularité qu'elle soit, serait susceptible d'être intégrée, reprise, discutée, amalgamée à un ensemble plus large, et continuellement transformée. On notera au passage que l'OA ainsi conçu revêt un sens élargi très voisin de la notion parente d'Open Source, propre à l'environnement du logiciel libre (le «code» n'étant pas propriétaire, il appartient à chacun de se le réapproprier suivant la pratique du «mash-up»), notion qui a d’ailleurs largement inspiré les militants du libre accès de la première heure et continue à servir de prisme intellectuel implicite chez quelques-uns de ses partisans.

Un tel modèle, qui va de pair avec un effacement progressif de la notion d'auteur, suppose l'abandon du droit d'auteur traditionnel et l'adoption d'un système de licences généralisé avec des droits de réutilisation optimums. Dans la mesure où cette vision fait toute sa place à la «science en train de se faire » au détriment d’une science établie et consacrée par la publication, il en résulte une forte ubiquité documentaire - le résultat important moins que le processus de la communication en lui-même, et la critique du résultat faisant partie intégrante de l'acte de recherche - de nature à compromettre toute forme d'évaluation bibliométrique stabilisée. Dès lors, c'est moins l'instrument bibliométrique qui est requis dans l'évaluation des contenus que la «popularité» de telle ou telle unité documentaire au sein d'une communauté, mesurée à l'aune de la fréquence des partages sur les réseaux sociaux ou du flux de commentaires dont elle fait l'objet ${ }^{13}$. Remarquons au passage

13 C'est tout le sens du manifeste «Altmetrics»: «With altmetrics, we can crowdsource peer-review. [...] Unlike the JIF [ie. Journal Impact Factor], altmetrics reflect the impact of the article itself, not its venue. Unlike citation metrics, altmetrics will track impact outside the academy, impact of influential but uncited work, and impact from sources that aren't peer-reviewed.»:

<http://altmetrics.org/manifesto/>. 
qu'il n'est pas sûr que l'amateur éclairé - figure souvent invoquée pour faire valoir les vertus du libre accès -, s'y retrouve, puisque l'échange peer-to-peer, de chercheur à chercheur, tend à promouvoir une communication délinéarisée et sans médiation, a priori réservée aux seuls initiés.

\subsection{D'UNE LOGIQUE DE CONTENU À UNE LOGIQUE DE SERVICES Le cas des «mega-journals»}

Sous leur forme paradigmatique, ces deux grandes virtualités de l'OA constituent des hypothèses purement théoriques qui ne s'opposent pas nécessairement de manière aussi schématique dès lors qu'il s'agit de penser les modalités concrètes d'implémentation du libre accès. Ainsi Stevan Harnad, inlassable promoteur des archives ouvertes, voit-il dans la solution de l'auto-archivage un objectif de moyen terme dont la finalité est explicitement le basculement du régime de la publication scientifique vers un modèle de publication financé en amont susceptible de favoriser l'émergence d'une offre d'accès centralisée aux documents publiés. La promotion des archives ouvertes n’a alors d'autre finalité que d'en arriver à une généralisation telle de la pratique de l'auto-archivage que les bibliothèques soient en mesure de résilier massivement leurs abonnements aux revues, sans pâtir d'un défaut d’accès préjudiciable à leurs usagers. Les crédits ainsi libérés pourraient être réorientés vers le paiement de droits à la publication plus équitables qu'ils ne le sont actuellement. La charge de la publication serait alors bien imputée à l'auteur - ou à son institution -, mais seulement après avoir été révisée à la baisse, de manière à refléter le coût réel d'une prestation minimale n'incluant plus la transformation éditoriale (laissée aux auteurs). De «fournisseur de contenus», l'éditeur est donc appelé, selon Stevan Harnad, à se transformer en «prestataire de services», mais d'un service borné à la gestion du peer-reviewing et à la fonction de labellisation scientifique. En l'absence de publication imprimée - dont le coût est trop important pour être supporté par les frais imputés aux auteurs -, la charge de l'édition en ligne, la gestion des accès et l'archivage numérique seraient alors mutualisés et reviendraient aux institutions publiques de la recherche. Notons à cette occasion que 
la disparition de la revue en tant que parution périodique (imprimée ou non) et vecteur d'un contenu éditorialisé est ici plus ou moins programmée.

Or c'est précisément une telle conversion des métiers de l'édition à une logique de services qui nous semble à l'œuvre à travers l'essor de formes éditoriales nouvelles, combinant verticalité et horizontalité, et participant d'une mutation profonde de la communication scientifique. Les «mega-journals $\gg^{14}$, dont PLoS One constitue aujourd'hui l'exemple le plus emblématique, sont ainsi devenus en quelques années des acteurs incontournables de la publication scientifique en libre accès dans le secteur des STM. Assumant pleinement leur rôle de prestataire de services, de telles plates-formes de publication se fondent sur le modèle économique «auteur-payeur» pour promouvoir une logique de diffusion purement numérique, sans contrainte en terme d'espace ni de périodicité. À la différence des revues scientifiques traditionnelles, ces «méga-revues » ne revendiquent aucune ligne éditoriale spécifique. Seul le critère disciplinaire au sens large faisant office de biais de sélection, elles tendent à couvrir un spectre très large de publications scientifiques : n'importe quel article relevant d'une discipline considérée comme pertinente est donc susceptible d’être publié, une fois validé sur la base de critères de scientificité a minima, et ce en flux continu. L'évaluation scientifique à proprement parler a lieu a posteriori sur la base de procédures ouvertes et transparentes (open peer-review), par l'ensemble de la communauté scientifique et au moyen d'outils collaboratifs. Ajoutons que dans le cas de PLoS One, la plate-forme a également mis en place des mesures bibliométriques article par article. Elle est ainsi l'exemple même d'une hybridation possible et réussie des fonctionnalités des deux paradigmes «légal-bibliométrique» et «collaboratif-en-réseau», empruntant au premier la logique de centralisation/standardisation des articles et au second les fonctionnalités sociales propres à la logique de réseau.

14 Des exemples de quelques-unes des principales méga-revues sont présentés ici: <http://openaccess.inist.fr/?Les-megarevues>. 
Avec un coût moyen de I 350 dollars par article et plus de 23000 articles déjà publiés, le succès - et la rentabilité - du modèle de PLoS One ne s'est en effet pas démenti, à ceci près que le facteur d'impact de la revue s'est progressivement dégradé ${ }^{15}$. De fait les revenus de la plate-forme ont été d’autant maximisés que le taux de rejet a lui-même été sensiblement abaissé (30\% contre plus de $70 \%$ pour une revue classique), portant nécessairement préjudice à la qualité éditoriale de l'ensemble et laissant dubitatif sur les conséquences d’une généralisation de ce type de modèle.

\subsection{D'UNE ÉCONOMIE DE RENTE À UNE ÉCONOMIE DE L'ATTENTION L'enjeu des données}

Outre le fait que le nouveau modèle qui tend à se faire jour n'est pas forcément moins lucratif que le système traditionnel de la souscription, il génère nécessairement un bruit documentaire croissant, et revient à déléguer le travail de sélection et de promotion des contenus à la seule puissance du réseau (algorithmes et partage). Ceci est de nature à introduire un certain nombre de biais amplificateurs (effet «mainstream», manipulations des taux, «buzz» sans rapport avec la qualité scientifique) entre autres effets pervers liés à la multiplication des revues en ligne et en libre accès ${ }^{16}$, à l'abaissement des taux de rejets et à la faible éditorialisation des contenus ${ }^{17}$.

15 Voir à ce sujet le billet de Phil Davis du 20 juin 2013 sur le blog The Scholarly Kitchen, «The Rise and Fall of PLOS ONE's Impact Factor $(2012=3.730) »$ : <http://scholarlykitchen.sspnet. org/2013/06/20/the-rise-and-fall-of-plos-ones-impact-factor-2012-3-730/>.

16 Ainsi, à côté de la multiplication des revues en ligne en libre accès, a-t-on vu se développer le phénomène des revues dites «prédatrices», publiant à la charge de l'auteur des articles dont la qualité ne fait l'objet d'aucune évaluation (voir, à ce sujet, l'article d'Hervé Morin dans Le Monde du 5 octobre 2013, «Édition scientifique: la preuve par le canular»: <http://lemonde.fr/idees/ article/2013//0/04/edition-scientifique-la-preuve-par-le-canular_3490120_3232.html>).

17 Dans un entretien publié par Richard Poynder, sur son blog, en date du 6 octobre 2013, Sami Kassab, analyste auprès du groupe Exane (BNP/Paribas) en charge des questions relatives aux médias et au secteur de la publication professionnelle, confirme cette tendance du «Gold OA» (auteur-payeur) à l'abaissement du taux de rejet en des termes dépourvus d'ambiguité: «in a Gold OA environment the lower price-per-article would be compensated for by the fact that these publishers would start publishing papers that historically they would have rejected: <http://poynder.blogspot.de/2013//0/media-research-analyst-at-exane-bnp.html>. 
Ce faisant, il s'agirait de prendre acte du fait que l'une des tendances déjà perceptibles en STM participe d'une mutation du régime de la communication scientifique et de l'évaluation de la recherche où le filtre à l'entrée (l'accès à la publication via la validation en amont par les pairs) le cèdera à terme à un filtre à la sortie, par le biais des outils bibliométriques et collaboratifs. Et en effet une telle tendance est sans doute inscrite dans les possibilités mêmes de la technologie numérique, qui consistent dans cette formule synthétique que nous empruntons aux auteurs d'un rapport sur le marché de l'OA : «empowering communication instead of allowing entrance» (Ricci et al., 20I3, p. 3I). On voit mal de ce point de vue ce qui justifierait encore de ne pas publier - si l'on veut bien entendre ce terme sous son acception symboliquement faible de « rendre accessible» - un résultat issu de la recherche publique, au motif qu'il n’aurait pas été préalablement évalué positivement. Et cela dans la mesure même où une simple validation préalable est de nature à permettre à l'évaluation scientifique de se produire dans des conditions de transparence optimales, en vertu des possibilités collaboratives du Web. Un tel bouleversement de la communication scientifique conduit nécessairement à une prolifération des biens d'information scientifiques qui n’est pas sans soulever quelques difficultés majeures.

Pour citer Philippe Aigrain, spécialiste du logiciel libre et militant du libre accès aux biens informationnels, dans un contexte de capacité d'attention décroissante: «It's not easier to have many people access for free a book, that it is to have many people access it by paying $\gg^{18}$. Rien n’aura donc été fait quand tout aura été rendu accessible librement, puisqu'un contenu en libre accès qui n’est pas promu dans un régime de surabondance documentaire (où la ressource rare n'est plus le contenu mais l'attention disponible du lecteur) est un contenu qui reste de facto quasi-inaccessible comme le montre la problématique du référencement dans Google. Dès lors, le libre accès généralisé ne garantirait pas que tous auront effectivement accès à tout,

I8 Propos tenus lors de la conférence «OA Monographs in the Humanities and Social Sciences Conference » (Jisc-Collections OAPEN Foundation) du I-2 juillet 2013, à la British Library de Londres. Les communications sont disponibles en ligne et celle de Philippe Aigrain (Panel Session: HSS

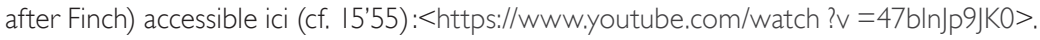


et un chercheur ne s'en trouvera pas moins dépourvu d'un accès à l'information pertinente devant pléthore d'informations peu ou mal discriminées.

Par delà leur logique respective, les différentes «voies» de l'OA font donc face à une problématique commune: comment produire de la rareté, condition de la réception scientifique, dans un environnement de surabondance documentaire et de baisse tendancielle de la qualité éditoriale? Que l'on se place dans l'un ou l'autre des deux paradigmes de la communication scientifique que nous avons évoqués («légal-bibliométrique» et «collaboratif-en-réseau»), l’ensemble du système ne tient finalement que par la capacité de certains à prescrire des contenus pertinents, en tirant pleinement partie des potentialités du numérique.

À cet égard, il n'est pas inutile de rappeler qu'une telle «économie de l'attention » n'est peut-être pas moins capitalistique que l'économie classique «de rente» (par souscription), comme en témoigne l'essor de nouveaux acteurs du type Academia, Mendeley, Researchgate, ou encore Mysciencework dans l'espace francophone, dont la fonction principale est de prescrire de la pertinence dans un contexte purement numérique ${ }^{19}$ : tirant partie de la dimension «communautaire» de la recherche et des fonctionnalités sociales du Web, ce type de plates-formes vise ainsi à capter l'attention canalisée par la concentration des ressources en libre accès, à des fins de monétisation de services associés ou de capitalisation des profils utilisateurs ${ }^{20}$. Un tel «capitalisme de la donnée » est d’ailleurs dénoncé avec vigueur par Pierre-Carl Langlais, auteur du blog Sciences communes, dans un texte très critique à l'endroit de la politique

19 On se reportera avantageusement à la synthèse très complète publiée par Aline Bouchard sur le blog de l'Urfist, le 14 février 2014, «Pour une utilisation critique des réseaux sociaux académiques», laquelle s'inquiète à cette occasion de «la part toujours plus importante des capitaux privés dans le monde académique»: <http://urfistinfo.hypotheses.org/2596>.

20 De ce point de vue, le libre accès peut faire le jeu d'intérêts capitalistiques tout aussi éloignés de l'idéal de partage de la connaissance que ceux qu'on prête à certains monopoles, à l'avantage toutefois d'acteurs «hors métier» et dont l'essentiel de l'activité économique réside dans la gestion des flux entrants et sortants - le buzz - et la monétisation des informations utilisateurs auprès d'agences publicitaires. La polémique récente autour des activités de la plate-forme Mysciencework, pourtant alliée au mouvement pour l'OA - et organisatrice de l'«Open Access Week» à Paris - témoigne sans doute de la prise de conscience d'un risque de privatisation à rebours du libre accès. Voir à ce sujet le billet de blog de Stéphane Pouyllau, du 30 octobre 2013, «Le libre accès privatisé» : <http://blog.stephanepouyllau.org/709>. 
commerciale d'Elsevier, qui y voit l'occasion d'un «profilage massif », tel que l'éditeur accomplirait une mutation profonde : «d'industrie culturelle classique du $\mathrm{XX}^{\mathrm{e}}$ siècle, il se métamorphoserait en industrie numérique sur le modèle de Google ou Facebook $»^{21}$.

Aussi, n’est-il pas certain qu’il découlerait automatiquement de la généralisation du libre accès un très grand progrès pour la communauté scientifique dans son ensemble si en définitive la domination sur le marché de la publication scientifique devait être dévolue aux seuls moteurs de recherche ou aux réseaux sociaux - et en général à tous les acteurs susceptibles de s'imposer comme prescripteurs (à commencer par les grands groupes d'édition traditionnels, seuls capables de s'adosser à une infrastructure de services complexe ${ }^{22}$ ).

Et ce pourrait être, in fine, l'un des effets collatéraux d'une telle économie de la publication scientifique, fondée essentiellement sur la sollicitation d'une attention qui n'est a priori pas disponible, que de participer d'un alignement des SHS sur le régime de publication en STM, avec l'adoption d'un format d’article standard, dans un souci de performance globale du système. Pareille conséquence avait d’ailleurs déjà été entrevue par Michel Pierssens (2007), dans un article du Débat intitulé Revue savantes : quel avenir? Commençant par remarquer que les sciences sociales s'efforcent depuis déjà longtemps de mimer les sciences exactes - tant dans le recours à l'anonymisation des évaluations que dans la multiplication des publications d’articles collectifs au détriment des monographies traditionnelles -, Michel Pierssens en vient à suggérer que l'un des avenirs possibles des humanités ou, en l'occurrence, des études littéraires, pourrait précisément passer par la transposition dans leur champ de ce qui s'impose depuis déjà longtemps chez les scientifiques : l'élimination de toute rhétorique, l'effacement des traces de la singularité de l'auteur et, avec elles, la disparition de l'auctoritas. «Pourquoi faire du style quand ce que le lecteur recherchera, c'est une

21 Notons que le libre accès ne contrevient nullement à une telle mutation, encouragée d'ailleurs par la logique bibliométrique. Voir le billet du 12 février 2014 «Faut-il signer l'accord Elsevier? 》 du blog Sciences communes: <http://scoms.hypotheses.org/l19>.

22 C'est ce dont prend acte Sami Kassab, dans l'entretien (déjà cité) réalisé par R. Poydner: «this is good news for large publishers, but bad news for smaller publishers, who could presumably expect to lose market share to their larger rivals. ») 
information, à la rigueur une idée, mais facilement condensée dans une ou deux phrases aussi frappantes que possible - ce que les résumés (abstracts) offrent déjà obligeamment et gratuitement » (ibid., p. I39). Il en résulterait alors une forme de standardisation des contenus visant avant tout l'efficacité, dans un contexte où prime l'utilitarisme en matière de recherche et où l'essentiel du discours scientifique est d’emblée véhiculé «par le biais de descripteurs ou de métadonnées indifférents à la politique éditoriale» (ibid., p. 138). Et l'auteur de soulever au passage une question très lourde de sens : «Dès lors, qu'importe qu'un article ait été choisi par une revue plutôt que par une autre?» (ibid.). Qui plus est, s'il n'est question que de valorisation des données et de résultats, quid d'un énième article de recherche sur Proust, par exemple, s'interroge encore l'auteur? La question est d'autant plus pertinente qu'en l'espèce, les seules «data» disponibles sont déjà en libre accès puisque le texte de la Recherche appartient au domaine public, en conséquence de quoi la mise en libre accès d'une nouvelle publication sur Proust est a priori, sauf contredit exceptionnel, sans «valeur ajoutée» par rapport au stock de connaissances déjà disponibles. Pour ne rien dire de son apport en terme d'innovation et de croissance...

C'est à ce stade du questionnement qu'il convient de rappeler que l'une des propriétés des SHS est précisément de n’être pas réductible au schéma général qui a court dans les sciences dites exactes - protocole/résultats/ validation -, de sorte qu'une partie non négligeable de leur intérêt passe au moins autant par la reformulation du connu dans des termes qui lui confèrent, à nouveaux frais, sens et intelligibilité, que par la production de données nouvelles. En l'absence d'un programme de recherche unifié susceptible de donner lieu à une logique véritablement cumulative, les SHS, quand même elles revendiqueraient une démarche quantitative, participent bien souvent d'une réactualisation des connaissances disponibles, voire d'une relecture dans des termes pertinents pour le contemporain des oppositions traditionnelles entre les quelques grands paradigmes établis. Ceci explique d’ailleurs sans doute la persistance de la forme monographique en SHS, dont l'«essai» demeure l'une des modalités d'expression privilégiées. À cet égard, l’idéal d'une science procédant par contributions successives, à l'image de Wikipedia 
ou du logiciel libre en général, est certes un idéal vertueux, porteur de grandes réalisations collectives, mais c'est un idéal qui rend peu justice à l'irréductibilité des points de vue et des options scientifiques et méthodologiques en SHS. Sauf à donner l'illusion que la science sociale serait enfin devenue une science expérimentale comme les autres (si tant est que la réciproque ne soit pas également vraie), la mise en pratique d’une «grande conversation scientifique » (pour reprendre l'image chère à Jean-Claude Guédon, autre illustre promoteur du libre accès) n'est pas sans se heurter à certaines limites inhérentes à la nature des sciences sociales, à commencer par l'attachement de bien des chercheurs à leur qualité d'auteur, ou au caractère éminemment instable et non-consensuel de la discussion scientifique à laquelle elles donnent lieu, à l'exemple de celle-là même qui nous occupe présentement.

\section{CONCLUSION \\ De l'avenir des revues scientifiques à comité de rédaction en SHS}

Dans une telle configuration de la communication scientifique, les revues traditionnelles en SHS, périodiques, imprimées ou non, sont-elles définitivement condamnées ou bien ont-elles encore un rôle à jouer? Nombreux sont ceux qui en doutent, tel Oliver Erztscheid (2012) qui voit dans les revues à comité de rédaction un objet «à bout de souffle», dépassé par une science 2.o, les réseaux sociaux, la communication en temps réel, le Big Data, etc. C'est cependant consommer peutêtre un peu vite l'épuisement de quelques-unes des fonctions propres aux revues traditionnelles - et qui pourraient s'avérer essentielles dans un contexte de surabondance documentaire - à savoir leur fonction de focalisation de l'attention, d'une part (un article qui paraît dans un numéro relève toujours de l' «événement», ce que n’est pas le dépôt dans un répertoire institutionnel, à plus forte raison si celui-ci est rendu obligatoire), et de polarisation de la recherche, d'autre part.

De fait, avant même d'être économique, la première barrière à l'accès est une barrière cognitive liée à la nécessité d’un apprentissage préalable de la méthode et des concepts qu'implique l'exploitation de toute ressource scientifique (ne serait-ce que la maîtrise de la langue de la publication) et au coût important, en terme d'investissement de temps et d'attention, que requiert la consultation 
approfondie de n'importe quelle littérature à prétention savante. De ce point de vue, le problème principal auquel sont confrontées les SHS n'est pas en premier lieu un problème d'accès aux ressources (déjà très abondantes en ligne) mais plutôt de faible demande à l'endroit de ces mêmes ressources, traduisant sans doute la persistance d'une barrière cognitive que le mouvement pour l'OA tend en général à négliger ${ }^{23}$. En levant la seule barrière économique (barrière levée de facto pour la quasi-totalité des chercheurs qui accèdent déjà aux principales ressources scientifiques par le biais des bibliothèques) [ce qui amène Hans Dillaerts a préférer l'expression d'« accès sans barrière» dans l'article qu'il publie dans ce dossier, section 3.3], l'on n’a ainsi rien fait pour faciliter la pénétration réelle des SHS dans la société, si l'on ne s'est pas donné les moyens, dans le même temps, d'en promouvoir l'apprentissage, d'une part, et d'en organiser l'exposition publique, d'autre part. Or ce sont précisément là deux des fonctions que le protocole traditionnel de la publication avait pour partie vocation à remplir et que les revues scientifiques perpétuent encore tant bien que mal. [Sur la fonction du protocole éditorial dans l'économie des marques et la valorisation apportée par les revues, voir l'article d'Olivier Bomsel, dans ce dossier, sections 3 et 6.]

À la différence des plates-formes de publications en ligne de type PLoS One, elles revendiquent encore une ligne éditoriale spécifique et continuent à jouer un rôle d'éditorialisation des contenus. Ce faisant, elles participent à l'animation d'une vie intellectuelle concrète en dessinant un paysage des sciences sociales diversifié, tout en créant les conditions d'une réception qui dépasse les limites de la seule communauté scientifique. Il se pourrait ainsi que l'on découvre sur le tard ce qui faisait le prix des revues traditionnelles en SHS - et qui ne peut être dissocié d'une certaine époque de la communication scientifique, où la diversité des publications était la manifestation concrète du pluralisme de la recherche en SHS -, à savoir leur fonction essentielle dans l'existence d'une vie intellectuelle et scientifique non unanime, en prise avec les enjeux de société et reflétant le caractère non consensuel du monde social.

23 Ce qui fait dire fort justement à Joseph Esposito que «the problem for many humanities publications is weak demand, not barriers to access ». Voir le billet du 28 janvier 2014 du blog The Scholarly Kitchen intitulé «The Market for Social Sciences and Humanities Publications »: <http://scholarlykitchen.sspnet.org/2014/01/28/the-market-for-social-sciences-and-humanities-publications/>. 
Si telle devait être la raison d'être des revues scientifiques en SHS, et s'il devait être fait obligation d'y renoncer faute de moyens, ou à l'avantage d'un système de communication scientifique faisant valoir le primat des «données » sur toute autre considération, alors le prix à payer pourrait s'avérer exorbitant pour la diversité des SHS et le pluralisme des points de vue sur le monde social.

Est-on sûr à cet égard d’avoir bien mesuré le sacrifice réel auquel il faut consentir, en termes de conventions éditoriales et scientifiques, pour parvenir à l'objectif qu'on s'est assigné ? Et n'est-on pas en train, en définitive, de casser l'outil de travail sans s'être au préalable mis d'accord sur ce qui sera appelé, à terme, à le remplacer? Est-on enfin certain qu’on pourra se porter garant dans la durée de la pérennité et de l'indépendance de ce par quoi on aura remplacé l'existant? Et tout cela ne vaut-t-il pas une messe, ou à tout le moins une étude d'impact préalable?

À l'aune de ce qui précède, on ne saurait en tout cas à se résoudre à borner le débat à une alternative simple. Les enjeux soulevés ici relèvent de choix indissociablement techniques et politiques qui requièrent un débat large et posé. Puisse ce dossier en être une convaincante illustration, autant qu'une invitation à poursuivre la discussion.

\section{BIBLIOGRAPHIE}

BARLUET S., 2005, «L'édition en histoire: anatomie d'une crise», Vingtième Siècle, p. $81-89$.

COLLECTIF, 2013a, «Open Access: le travail scientifique en sciences humaines et sociales et le débat public fragilisés par les mesures préconisées par la Commission européenne», Paris: <http://www.openaccess-shs.info/motion/>.

-, 2013b, «Qui a peur de l'Open Access», Le Monde, 15 mars: <http:// lemonde.frl sciences/article/20 I3/03//5/qui-a-peur-de-I-open-acces_|848930_|650684.html>.

COMMISSION EUROPÉENNE, 2012a, Recommandation de la Commission du 17.27.2012 relative à l'accès aux informations scientifiques et à leur conservation, Bruxelles: $<$ http://ec.europa.eu/research/science-society/document_library/pdf_06/ recommendation-access-and-preservation-scientific-information_fr.pdf>. 
-, 2012b, «Données scientifiques: le libre accès aux résultats de recherche stimulera la capacité d'innovation en Europe», Communiqué de presse, Bruxelles: <http://europa.eu/rapid/press-release_IP-12-790_fr.htm>.

DELVERT K., «La négociation de bouquets de périodiques électroniques: une négociation commerciale singulière », Négociations, 18-2, p. 7-23.

ERZTSCHEID O., 2012, «Science 2.0: renouveau de la recherche et/ou de l'échange scientifique?», Bibliothèques 2.0 à l'heure de média sociaux, Muriel Amar et Véronique Mesguich (éds), Paris, Éditions du Cercle de la Librairie, p. I59-[166] : <http://affordance.typepad.com/mon_weblog/2012/05/ science-20-renouveau-recherche-echange-scientifique.html>.

FINCH J. (et al.), 20I2, «Accessibility, sustainability, excellence: how to expand access to research publications», Working Group on Expanding Access to Published Research Findings: <http://www.researchinfonet.org/wp-content/ uploads/2012/06/Finch-Group-report-FINAL-VERSION.pdf>.

FIORASO G., 2013, «Discours de Genèviève Fioraso lors des $5^{e}$ journées Open Access » : <http://www.enseignementsup-recherche.gouv.fr/cid66992/ discours-de-genevieve-fioraso-lors-des-5e-journees-open-access.html>.

G8 SCIENCE MINISTERS STATEMENT, 20I3, Londres: <https://www.gov.uk/ government/news/g8-science-ministers-statement>.

MALOTAUX S., 20II, «Pour des bibliothèques engagées dans la diffusion des savoirs de l'université», BBF, 56-I, p. 54-59.

Nature, 2013, «The Future of Publishing», 495-7442: <http://www.nature.com/ news/specials/scipublishing/index.html>.

OCDE, 2005, «Digital broadband content: scientifique publishing»: Rapport DSTI/ICCP/IE(2004) II/FINAL: <http://www.oecd.org/internet/ieconomy/35393/45.pdf>.

-, 2007, Principes et lignes directrices de l'OCDE pour l'accès aux données de la recherche financée sur fonds publics: <http://www.oecd.org/fr/sti/scitech/38500823.pdf>. 
OFFICE OF SCIENCE AND TECHNOLOGY POLICY, 20I3, «Increasing Access to the Resultsof Federally Funded Scientific Research», Memorandum, Washington DC: <http://www.whitehouse.gov/sites/default/files/microsites/ ostp/ostp_public_access_memo_20|3.pdf>.

PIERSSENS M., 2007, «Revues savantes: quel avenir?», Le Débat, |45-3, p. 127-|40.

RCUK, 2013, RCUK Policy on Open Access and Supporting Guidance: <http://www.rcuk.ac.uk/documents/documents/RCUKOpenAccessPolicy.pdf>.

RICCI L., 20I3, Open Access: Market Size, Share, Forecast, and Trends, Outsell Inc.

SCHOPFEL J. et al., 2012, «Éfficience et économie d'échelle. Une étude sur l'édition de revues en libre accès», Études de communication, 38-I, p. 167-185.

SCIENCE EUROPE POSITION STATEMENT, 2013, Principles for the Transition to Open Access to Research Publications, Bruxelles:

<http://www.scienceeurope.org/uploads/Public\%20documents\%20and\%20 speeches/SE_OA_Pos_Statement.pdf>.

Tracés, 2013, «Open access: la schizophrénie française», Le carnet de Tracés, 8 mars : <http://traces.hypotheses.org/805>.

VAJOU M. et al., 2009, «Les enjeux économiques de l'édition scientifique, technique et médicale: analyses et questions clés», Les Cahiers du numérique, 5-2, p. 143-172. 
